\title{
Seeking in Data an Answer to the Question of Empowerment
}

\author{
Anthony Elliott*
}

Delving into data can be by turns demanding, disconcerting, destabilizing and oftentimes, frankly, dangerous. Such conundrums, raised to the second power in the age of advanced artificial intelligence, arise from the complex antagonism between the thirst for data demonstrated by capitalist enterprises and the desire for privacy demonstrated time and again by consumers. Another way of putting this point, one more in keeping with the ideological and technological discourses of neo-liberalism, is the wellknown paradox between data sharing and data privacy. Data sharing has generated fundamental issues of data boundaries and non-controllability of data and concomitantly rendered privacy and security issues increasingly prominent.

In a brilliant recent theoretical analysis, the Italian philosopher Massimo Durante casts the automated capture of data as a microcosm of the progressive adaptation of the environment and transformation of the world created by "unique computational power". ${ }^{1}$ For Durante, computational power refers not simply to the vast spread of data processing capabilities resulting from the digital revolution, but to deep changes in structures of social action, world adaptation as well as representations of reality. Each is an indispensable condition of the other, and importantly these transformations go largely unnoticed in the routines of everyday life. For Durante, trust undergoes profound mutation as a consequence of computational power. We are today, increasingly, resigned to trusting the complex computational systems which power up our lives. According to Durante, three key issues arise: "1) Informationally: to what extent are we able to know and measure the computational system's trustworthiness; 2) Environmentally: to what extent do we share common concerns or purposes with these computational systems?; 3) Normatively: how can we prompt or appraise the computational system's loyalty?". ${ }^{2}$ To begin to address these issues, Durante contrasts what he calls intersubjective trust and systemic trust. The intersubjective scoops up the conduct of trusting interactions with various artificial agents; this speaks to the investment of emotion which humans transfer to non-human others - such as new digital technologies and automated intelligenct machines. Systemic trust has to do with, among other things, Al-enabled digital

\section{DOI: $10.21552 / \mathrm{edpl} / 2021 / 2 / 4$}

* Professor Anthony Elliott is Dean of External Engagement, Professor of Sociology and Executive Director of the Hawke EU Jean Monnet Centre of Excellence and Network at the University of South Australia. Professor Elliott is also Global Professor of Sociology (Visiting) in the Graduate School of Human Relations, Keio University, Japan and Visiting Professor of Sociology at University College Dublin, Ireland. For Correspondence: <Anthony.Elliott@unisa.edu.au> 
technologies that provide a great deal of the essential security which underpins the system coordination of day-to-day social life. "The tendency to specifically trust this or that algorithm, this or that artificial agent, this or that technological device", writes Durante, "gives way to the tendency to generally trust the digital world, where the environment and the representation of reality will gradually be adapted to the functioning of technologies". ${ }^{3}$ Women and men increasingly entrust expert computational systems and artificial agents to decide or act on their behalf, but in the process fear that they are becoming disengaged from their own lives, the lives of others and wider public life.

Like the best of scholarly works seeking to invigorate self-reflection and enrich public political debate in times of mixed signals and elusive understanding, the contributions in this volume seek to extend the process of reinterpretation of social, cultural and political concerns for individuals from privacy and data protection perspectives. The contributions can be read in many ways - just as the mixed signals from our "culture of $\mathrm{Al}^{\prime \prime}$ can be interpreted in many ways. ${ }^{4}$ Personally, I particularly value Mariam Hawath's attempt to interrogate new measures to extend existing policy solutions which "enhance protection afforded to data subjects who cannot successfully exercise control over automated decision-making". Or, equally illuminating is Christof Koolen's proposals to advance higher levels of informational protection for consumers in data-driven smart environments.

Considerations of this kind are vital. Awareness that cultural ambiguity is endemic and incurable across the culture of Al might, however, also facilitate a somewhat different approach to these major issues of our times and our lives in these times - as Durante understands only too well. Too many current academic and policy proposals as currently formulated are scarcely adequate to confront the twin challenges of digital empowerment and technological displacement. If we wish to adequately understand this challenge, it is critical to grasp that the empowerment of individuals (as workers, employees, citizens) is interwoven with a broader set of social changes than is captured under demands for the acquisition of data protection alone. As Zygmunt Bauman argues, "to be 'empowered' means to be able to make choices and act effectively on the choices made, and that in turn signifies the capacity to influence the range of available choices in the social settings in which choices are made and pursued". ${ }^{5}$ These broad lines of economic and social challenge identified by Bauman provide some insight into the daunting problems facing governments and citizens alike. Empowerment in the context of the culture of Al cannot only be limited to demands for more privacy, more protections and more transparency; empowerment must instead encompass the development of genuine capabilities, capacities and resources at the level of individuals to make decisions, and influence outcomes, about the place of big data in our lives and our lives in these times.

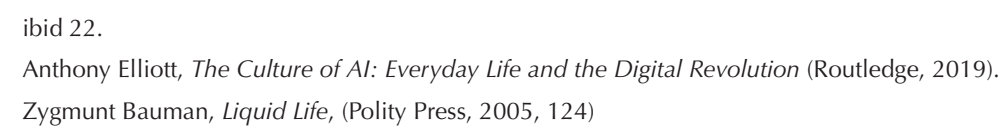

